\title{
Modulation of HLA-DR in dry eye patients following 30 days of treatment with a lubricant eyedrop solution
}

This article was published in the following Dove Press journal:

Clinical Ophthalmology

24 June 2015

Number of times this article has been viewed

\section{Karen B Fernandez \\ Seth P Epstein \\ Geoffrey S Raynor \\ Alan T Sheyman \\ Morgan L Massingale \\ Peter G Dentone \\ Lukas D Landegger \\ Penny A Asbell}

Department of Ophthalmology, Mount Sinai School of Medicine, New York, NY, USA
Correspondence: Penny A Asbell Department of Ophthalmology, Mount Sinai School of Medicine, I Gustave L. Levy Place, Box II83, New York, NY 10029, USA

$\mathrm{Tel}+|21224| 79||$

Fax $+|2| 224 \mid 4550$

Email penny.asbell@mssm.edu
Purpose: To determine the changes in dry eye disease (DED) severity and the percentage of cells expressing HLA-DR on the ocular surface following treatment with lubricant eyedrops containing polyethylene glycol and propylene glycol (PEG/PG) and the gelling agent hydroxypropyl guar (HP-Guar).

Patients and methods: Nineteen patients with DED used PEG/PG + HP-Guar eyedrops four times per day for 30 days. Assessments included DED severity (Ocular Surface Disease Index [OSDI], corneal staining, conjunctival staining, tear film break-up time [TFBUT], and Schirmer testing) and impression cytology of the conjunctiva with masked flow cytometry at baseline and at 30 days.

Results: There was a significant decrease in corneal staining $(P<0.01)$, OSDI $(P=0.02)$, and TFBUT $(P<0.01)$ following treatment with PEG/PG + HP-Guar. Results from flow cytometry revealed a significant decrease in cells expressing $\operatorname{HLA}-\mathrm{DR}(P=0.02)$.

Conclusion: Treatment with PEG/PG + HP-Guar eyedrops showed improvement in dry eye severity and reduction in surface inflammation as indicated by a reduction in HLA-DR expression.

Keywords: artificial tears, dry eye disease, hydroxypropyl guar, inflammation, lubricating eyedrops, polyethylene glycol, propylene glycol

\section{Introduction}

Dry eye disease (DED) is a medical condition associated with symptoms of ocular irritation, visual disturbance, and tear film instability, often going hand in hand with ocular surface damage and inflammation. Dry eye is considered a multifactorial disease with numerous causes and risk factors. ${ }^{1}$ Some clinicians may differentiate DED into an aqueous deficient type (aqueous deficient DE) or an evaporative type (evaporative $\mathrm{DE}$ ), although most of the time, the distinction is not that clear. However, whether aqueous deficient or evaporative, both can result in a cycle of inflammation, tear hyperosmolarity, and ocular surface damage. ${ }^{1}$ Inflammation has a key role in DED and is both a result and cause of tear deficiency and ocular surface damage.

Although common, diagnosis and clinical evaluation of dry eye typically rely on patient symptoms and clinician's subjective grading of ocular surface staining patterns. Monitoring of treatment efficacy is mostly dependent on improvement of patient symptoms, as well as signs of ocular surface damage. ${ }^{1,2}$

The conjunctival epithelium has an important role in the ocular surface defense mechanism. Aside from it being an anatomical barrier to the external environment, the conjunctival epithelium has the ability to build up an inflammatory response by 
promoting leukocyte migration by expressing cell adhesion molecules, such as intercellular adhesion molecule 1, and major histocompatibility complex II molecules, such as human leukocyte antigen D-related (HLA-DR), on antigenpresenting cells. HLA-DR plays an important role in T-cell activation and is overexpressed in many patients with DED. ${ }^{3-7}$ HLA-DR expression on surface cells has been shown to correlate with inflammation and was found to be increased in patients with dry eye. Flow cytometric analysis of samples taken from the ocular surface by impression cytology that appropriately stained may be used to detect this proinflammatory marker. ${ }^{8-14}$ Because HLA-DR is considered a biomarker for the increased inflammation on the ocular surface associated with DED, detection of HLA-DR by flow cytometric analysis may be a useful indicator for identifying ongoing inflammatory processes, classifying the severity of DED, and evaluating the response to treatment. Therefore, the relative percentage of cells obtained from impression cytology, which highly express HLA-DR, may provide a minimally invasive objective measure of inflammation of the ocular surface.

The test treatment used in this study was a lubricant eyedrop product containing polyethylene glycol (PEG), propylene glycol (PG), and hydroxypropyl guar (HP-Guar) preserved with polyquaternium-1 (POLYQUAD $\left.{ }^{\circledR}\right)$. The preservative polyquaternium-1 has been shown in animal studies to be less toxic to the ocular surface than the traditional preservative benzalkonium chloride. ${ }^{15-19} \mathrm{HP}$-Guar effectively relieves signs and symptoms related to moderate dry eye, with measurable improvements evident in both objective staining and subjective questionnaire. ${ }^{19}$

The purpose of this study was to evaluate the efficacy of this lubricant eyedrop solution in improving signs and symptoms of DED, as well as its influence on surface inflammatory markers measured by flow cytometry.

\section{Materials and methods}

\section{Study design}

This was a single-arm, open-label study of an over-the-counter lubricant eyedrop with active ingredient PEG/PG + HP-Guar (www.ClinicalTrials.gov identifier, NCT00946777). This Institutional Review Board-approved trial was conducted in accordance with the Good Clinical Practice guidelines for the evaluation of medical products and following the tenets of the Declaration of Helsinki.

\section{Patients}

Patients were selected from those being seen at the Faculty Practice Associates at the Department of Ophthalmology,
Mount Sinai School of Medicine. Nineteen patients who were diagnosed with mild to moderate DED with typical signs and symptoms were recruited. All patients read, understood, and signed the informed consent form before any examination was obtained. Male as well as female patients who were 18 years of age or older were eligible for the study. Both eyes of each patient were included in the trial.

The major inclusion criteria were at least one symptom of dry eye that was graded moderate to severe (rated on a 4-point scale) or clinical evidence of dry eye by Schirmer testing $(<10 \mathrm{~mm} / 5 \mathrm{~min})$. Patients were required to show evidence of ocular surface disease, moderate vital staining of the ocular surface with fluorescein (for corneal evaluation) and lissamine (for conjunctival evaluation), and had to be willing to discontinue current eye medications, use PEG/ PG + HP-Guar four times a day, and return on follow-up dates throughout the course of the study. Patients with any of the following were excluded from the study: uncontrolled systemic (ie, diabetes mellitus, rheumatoid arthritis, and other immunologic or autoimmune diseases) or ocular disease (ie, glaucoma or uveitis), dry eye due to seasonal allergic conjunctivitis, contact lens-related conjunctivitis or other acute or seasonal diagnoses, monocular and/or legal blindness, history of ocular surgery or trauma within the last 6 months, or participation in an ophthalmic drug or device research trial within 30 days before entry in this study.

\section{Materials}

US Food and Drug Administration-approved over-thecounter lubricant eyedrops were used in this study. The test solution was a PEG/PG-based, HP-Guar-containing lubricant eyedrop, preserved with POLYQUAD ${ }^{\circledR}$ (Systane Ultra $^{\circledR}$ Lubricant Eye Drops, Alcon Laboratories, Inc., Fort Worth, TX, USA). The product was supplied in its original, unopened container.

\section{Procedures}

After the study was explained and consent given, eligibility criteria were assessed to ensure all patients had dry eye signs and symptoms. After baseline screening and baseline impression cytology, all 19 patients were instructed to discontinue other artificial tear products but could continue use of Restasis ${ }^{\circledR}$ (cyclosporine A; Allergan, Inc., Irvine, CA, USA) if they had been using it for at least 6 months, because baseline readings may change significantly if chronic usage was discontinued. They were given PEG/PG + HP-Guar, to be used four times per day to both eyes for 1 month. At the end of 1 month, clinical assessments were performed 
to compare before and after treatment. Samples taken by impression cytology were analyzed by a masked examiner, using flow cytometry. Inflammatory markers (percent cells highly expressing HLA-DR) before and after study treatment were determined.

\section{Impression cytology}

Cells were collected via the technique adapted from previously established methods. ${ }^{4,8,12}$ In brief, after administration of a single drop of topical anesthetic $(0.5 \%$ proparacaine hydrochloride ophthalmic solution), two sterile $0.20-\mu \mathrm{m}, 13-\mathrm{mm}$ polyether sulfone filter membranes (Supor-6-Membranes, Pall Life Sciences, Ann Arbor, MI, USA) were applied bilaterally onto the superior bulbar and supero-temporal aspects of the conjunctiva without applying pressure, at least $15 \mathrm{~min}$ utes after any prior ophthalmic procedure(s). Cells were collected in a sterile fashion and placed in $2 \mathrm{~mL}$ paraformaldehyde $(0.05 \%$ in phosphate-buffered saline [PBS; Mediatech Inc, Manassas, VA, USA]) in $5 \mathrm{~mL}, 12 \mathrm{~mm} \times 75 \mathrm{~mm}$ test tubes (BD Biosciences, Franklin Lakes, NJ, USA) for direct immunofluorescence and subsequent cell cytology. All specimens from a given eye were placed into a single test tube. Samples from contralateral eyes were placed into separate tubes. The tubes were labeled with only a masked code number. They were then stored at $4^{\circ} \mathrm{C}-8^{\circ} \mathrm{C}$ in the dark until staining. One to two drops of prophylactic antibiotics ( $0.5 \%$ moxifloxacin hydrochloride) were administered to both eyes after collection.

\section{Immunostaining}

The percentage of highly HLA-DR-positive (inflammatory) cells was calculated by direct immunofluorescent staining followed by cell cytology. ${ }^{4,8,12}$ In brief, upon initiation of staining, the samples were diluted with $2 \mathrm{~mL}$ cold $\left(4^{\circ} \mathrm{C}-8^{\circ} \mathrm{C}\right)$ $0.5 \%$ bovine serum albumin (BSA) in PBS. Cells were separated from the filters by mechanical agitation (vortex; 15 seconds) followed by centrifugation at 1,600 rpm for 5 minutes $\left(4^{\circ} \mathrm{C}\right.$; Beckman GS 6R Centrifuge, Beckman Coulter, Inc, Fullerton, CA, USA). The filters were removed, the cells resuspended $(0.5 \% \mathrm{BSA}$ in PBS), and $40 \%$ of the resulting samples split into new test tubes to be used as negative controls. The cells of all tubes were concentrated by recentrifugation $\left(1,600 \mathrm{rpm}, 5\right.$ minutes, $\left.4^{\circ} \mathrm{C}\right)$ followed by decanting and resuspension in $200 \mu \mathrm{L}(0.5 \%$ BSA in PBS). Samples were stained for HLA-DR using a direct immunofluorescent technique. Fluorescein isothiocyanateconjugated monoclonal mouse antihuman HLA-DR, alpha chain clone, TAL.1B5 antibody (BD Biosciences) and fluorescein isothiocyanate-conjugated mouse antihuman $\mathrm{IgG}_{2 \mathrm{~b}}$ (negative isotypic control; BD Biosciences) were diluted 1:50 in 0.5\% BSA in PBS and were incubated with samples at $4{ }^{\circ} \mathrm{C}$ in the dark. Samples were washed $(0.5 \% \mathrm{BSA}$ in PBS) twice after centrifugation $(1,600 \mathrm{rpm} \times 5$ minutes, $4^{\circ} \mathrm{C}$ ) and resuspended in $400 \mu \mathrm{L}$ BSA $(0.5 \%)$ in PBS in preparation for flow cytometry. ${ }^{4,8,12}$

\section{Flow cytometry}

After immunostaining, cells in the resulting solutions were analyzed by flow cytometry (FACS-Calibur, BD Biosciences), equipped with an argon ion laser, which emits at $488 \mathrm{~nm}$. Preliminary calibrations were made using immortalized conjunctival epithelium (clone 1-5c-4 [WongKilbourne derivative (D) of Chang conjunctiva], ATCC ${ }^{\circledR}$, CCL-20.2 $\left.2^{\mathrm{TM}}\right)^{20,21}$ as the negative control ${ }^{22}$ and HTK-H cells (human corneal fibroblast cell line) $)^{23}$ as the positive control for HLA-DR staining.

To analyze, dot plots for cell size (forward scatter) versus cellular granularity (side scatter) and fluorescence channel (FL) 1 versus cellular granularity (side scatter) and a histogram (FL1 versus event count) were plotted, demonstrating a single homogenous cell population (Figure 1). Analytic gates were set around the area of interest to exclude cellular debris and aggregates. The percentage of HLA-DR-positive cells was obtained from a cytogram, yielding the histogram's statistics, indicating the mean intensity of cell fluorescein that was proportional to HLA-DR expression. In each sample, 1,000-10,000 cells were analyzed; samples containing less than 1,000 cells were discarded. Samples from each eye were

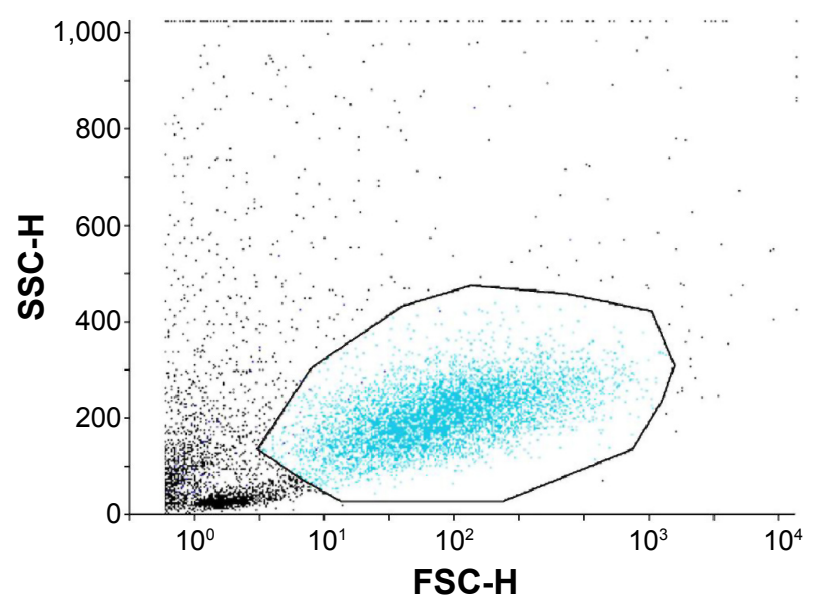

Figure I Representative dot plot of impression cytology samples.

Notes: Cell size (forward scatter pulse height [FSC-H]) versus cellular granularity (side scatter pulse height [SSC-H]) data of all events collected by cell cytology are plotted. The outlined area demonstrates a single homogenous cell subpopulation. In each sample, I,000-10,000 cells were analyzed; samples containing less than I,000 cells were discarded. All specimens were examined by a masked observer. 
analyzed separately and independently. ${ }^{8,9,11-14}$ All specimens were processed by a masked observer.

\section{Clinical assessments}

Clinical assessments included the Ocular Surface Disease Index (OSDI) questionnaire, tear film break-up time (TFBUT), corneal and conjunctival staining with fluorescein and lissamine, and Schirmer testing with anesthesia. ${ }^{24-27}$ The OSDI questionnaire was completed following the standard technique by Stevenson et al. ${ }^{27}$ Standardized TFBUT was done by instillation of $5 \mu \mathrm{L}$ of $2 \%$ fluorescein onto the conjunctival fornices and recording the time for the tear film to break up on the corneal surface. The average of three measurements was recorded. Corneal staining was then assessed using the National Eye Institute scale for grading, dividing the corneal surface into five areas and scoring accordingly (grade of $0-15$ per eye). ${ }^{24-26}$ Conjunctival staining was evaluated using lissamine-impregnated strips wetted with artificial tears, and conjunctival staining pattern was graded according to density, using a scale of $0-3$ on both the nasal and temporal conjunctiva (grade of 0-6 per eye). ${ }^{24,25}$ Impression cytology was then performed using the technique described earlier. Schirmer testing with anesthesia was performed by instilling anesthetic drops onto the lower conjunctival fornices, then waiting for 3 minutes before placing the tear strips on the lower lid margin. Patients were asked to close their eyes for 5 minutes before taking the readings. ${ }^{24}$

The modified Dry Eye WorkShop (DEWS) score was graded based on the aforementioned tests and clinical evaluation mentioned. The patients were then given a DEWS classification of DED severity (Table 1), where scores from individual dry eye test were replaced with severity grading score of $1-4 .{ }^{24}$ Visual acuity, intraocular pressure measurement, and slit lamp biomicroscopy were also done as part of safety parameters.

\section{Statistical analysis}

Statistical analysis was performed using SPSS (IBM Corporation, Armonk, NY, USA) and NCSS (NCSS, LLC, Kayesville, UT, USA). Within-patient, before and after treatment comparisons were conducted using paired $t$-tests. All tests were two-sided with a confidence level set to $95 \%$.

\section{Results}

A total of 19 patients were enrolled in the study. Three patients did not return for their day 30 evaluation and were not included in the final analysis. Therefore, 16 patients or 32 eyes were evaluated (Table 2). There were two enrolled patients who had been on Restasis ${ }^{\circledR}$ for more than 6 months.

After 30 days of treatment with PEG/PG + HP-Guar, statistically significant changes $(P<0.0001)$ were observed in the mean corneal staining ( $5.38 \pm 3.63$ to $2.71 \pm 3.08$ points), with an improvement of 2.68 points or $51 \%$; TFBUT (2.39 \pm 1.64 to $4.19 \pm 2.25$ points), with an improvement of 1.80 points or $115 \%$; and the total DEWS score ( $2.10 \pm 0.90$ to $0.82 \pm 0.83$ points), with an improvement of 1.28 points or $58 \%$. There were also statistically significant changes in mean OSDI score (41.54 23.62 to $32.91 \pm 23.16$ points), with an improvement of 8.63 points or $19 \%$ ( $P=0.0204)$. Results from conjunctival staining showed improvements after 30 days of treatment; however, they were not statistically significant (Table 3 ).

Sixty-three percent of patients had decreased HLA-DR expression in one or both the eyes, and $43 \%$ had increased expression in one or both the eyes after 30 days of lubricant eyedrop use. The percentage of HLA-DR-positive cells obtained from the resultant cytogram, indicating the mean intensity of cell fluorescein, which was proportional to HLA-DR expression, revealed a change from $7.17 \pm 6.10$ to $3.77 \pm 2.12$, showing a significant decrease of about $50 \%$ ( $P=0.0212$; Table 4).

These numbers reflect a general decrease in the inflammatory marker HLA-DR in eyes treated with PEG/PG + HP-Guar

Table I Modified Dry Eye WorkShop scale with numeric range

\begin{tabular}{|c|c|c|c|c|}
\hline \multirow[t]{2}{*}{ Assessment } & \multicolumn{4}{|c|}{ DED severity ${ }^{\mathrm{a}}$} \\
\hline & Grade I & Grade 2 & Grade 3 & Grade 4 \\
\hline Discomfort severity and frequency, OSDI score & $<15$ & $16-30$ & $31-45$ & $>45$ \\
\hline Tear film break-up time, ${ }^{\text {b }}$ seconds ${ }^{24}$ & $15-18$ & $<10$ & $<5$ & Immediate \\
\hline Schirmer testing, ${ }^{\mathrm{c}} \mathrm{mm}^{24}$ & $10-15$ & $<10$ & $<5$ & $<2$ \\
\hline Corneal staining score, ${ }^{d} \mathrm{NEI}$ scale ${ }^{22,24}$ & $0-3$ & $\mathrm{I}-8$ & $9-14$ & $14-15$ \\
\hline Conjunctival staining score $\mathrm{e}^{\mathrm{e}, 23,24}$ & $0-1$ & $2-3$ & $4-5$ & 6 \\
\hline
\end{tabular}

Notes: aSeveral measurements were considered before categorizing patients into an overall severity classification. Eyes were measured individually and a grade of 0 was given if, in the opinion of the clinician, the patient had negligible signs and symptoms of DED. ${ }^{\mathrm{b}} \mathrm{Tear}$ film break-up time represents an average of three separate readings. ${ }^{\mathrm{C}} \mathrm{Distance}$ assessed after 5 minutes. ${ }^{d} \mathrm{NEI}$ scale range, 0-15 points. eScore range, 0-6.

Abbreviations: DED, dry eye disease; NEI, National Eye Institute; OSDI, Ocular Surface Disease Index. 
Table 2 Patient demographics

\begin{tabular}{ll}
\hline Feature & Patients, $\mathbf{n}(\%)$ \\
\hline Patients analyzed $^{\mathrm{a}}$ & $16(84)$ \\
Mean \pm SD age, years $^{\mathrm{b}}$ & $61.29 \pm 16.44$ \\
Sex & \\
$\quad$ Female & $11(68)$ \\
$\quad$ Male & $5(32)$
\end{tabular}

Notes: ${ }^{a}$ Data reflect the percentage of patients enrolled $(n=19)$. ${ }^{b} D a t a$ reflect patients analyzed.

for 4 weeks. Representative plots from one patient (taken from the right eye) are shown, demonstrating HLA-DR-positive cells before and after treatment (Figures 2 and 3 ).

There were no reports of adverse events for the duration of the study, neither observed by the investigator nor reported by the patient.

\section{Discussion}

Our study suggests that regular use of over-the-counter lubricant eyedrops for 1 month may lead to decreased inflammatory response of the ocular surface, as measured by the percentage of HLA-DR-positive cells obtained through impression cytology. This study also suggests a mechanism of how a lubricant eyedrop solution may help in providing improvement in DED beyond palliative treatment. In addition, it demonstrates the usefulness of a biomarker of inflammation, percent-positive HLA-DR cells, for assessing treatment in DED and inflammation of the ocular surface and its responsiveness to change with an intervention. The use of minimally invasive objective metrics for evaluating the severity of disease and effectiveness of treatment may provide better endpoints for future clinical trials of DED and other ocular surface abnormalities.

Inflammation of the ocular surface plays a vital role in DED. Levels of proinflammatory cytokines, chemokines, and inflammatory $\mathrm{T}$ cells have been found to be elevated not only in patients suffering from autoimmune ocular diseases such as Sjögren's syndrome but also in non-Sjögren's dry eye. Stability of the ocular surface depends on a number of factors interplaying to keep and preserve the integrity of the tear film and transparency of the cornea. These factors include sensory inputs from the ocular surface resulting in reflex tear production as well as proper tear film composition. If any of these is altered, inflammation will result, causing even more tear film instability, entering into a vicious cycle. ${ }^{1-7}$

HLA-DR is a glycoprotein transmembrane complex, part of the major histocompatibility complex II. These molecules are present in antigen-presenting cells, which activate $T$ cells as part of an inflammatory cascade. Although HLA-DR is also expressed in normal conjunctiva, studies have shown that expression is significantly upregulated in patients with DED compared with normal eyes. ${ }^{3-7}$

Alterations of the conjunctival surface can be analyzed by impression cytology. This is a minimally invasive method wherein surface epithelial cells can be harvested and analyzed using indirect immunofluorescence and flow cytometry. It was believed that only immune cells express HLA-DR, but using the techniques mentioned earlier in the analysis of dry eye patients, studies have shown the expression of HLA-DR by conjunctival epithelial cells is correlated with the severity of the disease. ${ }^{6}$ This marker appears to reflect disease progression in mild to moderate dry eyes and may even be expressed in those with mild disease with no signs of ocular surface damage. ${ }^{5}$ It would then theoretically follow that the use of immunomodulatory agents had a role in the treatment of dry eyes, which is the reason why a wide range of drugs (eg, corticosteroids, doxycycline, cyclosporine A, mycophenolate mofetil, tacrolimus, rapamycin, leflunomide) have been tested in laboratory or clinical settings for the treatment of DED.

Table 3 Severity of dry eye symptoms at baseline and after 30 days of using PEG/PG lubricant eyedrops four times daily

\begin{tabular}{|c|c|c|c|c|c|c|c|}
\hline \multirow[t]{2}{*}{ Variable $^{a}$} & \multicolumn{3}{|c|}{ Baseline } & \multicolumn{3}{|c|}{ Day 30} & \multirow[t]{2}{*}{$P$-value ${ }^{c}$} \\
\hline & $\mathbf{n}^{\mathbf{b}}$ & Mean (SD) & Range & $n^{b}$ & Mean (SD) & Range & \\
\hline DEWS score & 32 & $2.10(0.90)$ & $0-4$ & 32 & $0.82(0.83)$ & $0-2$ & $<0.0001$ \\
\hline OSDI score & 16 & $41.54(23.62)$ & $10-93.75$ & 16 & $32.91(23.16)$ & $4.55-91.66$ & 0.0204 \\
\hline Conjunctival staining & 32 & $0.56(0.88)$ & $0-3$ & 32 & $0.24(0.50)$ & $0-2$ & 0.1057 \\
\hline Corneal staining & 32 & $5.38(3.63)$ & $0-13$ & 32 & $2.71(3.08)$ & $0-12$ & $<0.0001$ \\
\hline Tear film break-up time, seconds & 32 & $2.39(1.64)$ & $0.90-9.67$ & 32 & $4.19(2.25)$ & $1.29-11.89$ & $<0.0001$ \\
\hline Schirmer testing, mm & 32 & |3.44 (7.8I) & $4-35$ & 32 & $12.82(6.80)$ & $4-35$ & 0.5859 \\
\hline$\%$ HLA-DR+ & 32 & $7.17(6.10)$ & $1.07-13.27$ & 32 & $3.77(2.12)$ & $0.70-8.97$ & 0.0212 \\
\hline
\end{tabular}

Notes: aPotential score ranges: DEWS score, 0-4; OSDI score, 0-100; conjunctival staining score, 0-6; corneal staining score, 0-15. 'bample sizes reflect number of eyes for all assessments other than the OSDI questionnaire, which was based on number of patients. ${ }^{c} P$-values reflect results of paired $t$-tests for within-patient comparisons of assessments at baseline versus day 30 . Statistical significance is indicated with bold text.

Abbreviations: DEWS, Dry Eye WorkShop; HLA-DR, human leukocyte antigen D-related; OSDI, Ocular Surface Disease Index; PEG, polyethylene glycol; PG, propylene glycol. 
Table 4 Histogram \% gated cells at baseline and after 30 days of using PEG/PG lubricant eyedrops four times daily

\begin{tabular}{|c|c|c|c|}
\hline \multirow{2}{*}{$\begin{array}{l}\text { Sample } \\
\text { number }\end{array}$} & \multicolumn{2}{|c|}{ \% HLA-DR (OD/OS) } & \multirow{2}{*}{$\begin{array}{l}\text { Days between } \\
\text { visits }\end{array}$} \\
\hline & Baseline & Day 30 & \\
\hline 001 & $4.14 / 2.75$ & NA & NA \\
\hline 002 & $3.87 / 3.12$ & $5.05 / 2.89$ & 31 \\
\hline 003 & $9.61 / 13.17$ & NA & NA \\
\hline 004 & I I.07// 2.87 & I.I2/2.95 & 34 \\
\hline 005 & $23.21 / 21.13$ & $4.26 / 0.70$ & 34 \\
\hline 006 & $20.88 /|I .4|$ & $1.11 / 1.81$ & 28 \\
\hline $007^{a}$ & $7.31 / 3.29$ & $1.45 / 2.30$ & 36 \\
\hline 008 & $0.58 / 0.35$ & $8.52 / 8.97$ & 43 \\
\hline 009 & $0.64 / 0.63$ & $4.34 / 4.29$ & 26 \\
\hline 010 & $2.02 / 1.18$ & $3.23 / 2.57$ & 32 \\
\hline 011 & $4.60 / 10.04$ & $6.31 / 5.12$ & 24 \\
\hline 012 & $13.61 / 4.80$ & NA & NA \\
\hline $013^{a}$ & $3.09 / 5.59$ & $1.23 / 4.96$ & 41 \\
\hline 014 & $7.95 / 17.55$ & $3.38 / 2.78$ & 30 \\
\hline 015 & $4.76 / 12.19$ & $2.91 / 2.59$ & 28 \\
\hline 016 & $6.54 / 7.72$ & $3.87 / 2.40$ & 36 \\
\hline 017 & $1.44 / 2.58$ & I.48/7.17 & 35 \\
\hline 018 & $6.12 / 3.59$ & $4.30 / 6.65$ & 28 \\
\hline 019 & $5.50 / 1.66$ & $5.23 / 4.76$ & 30 \\
\hline Mean \pm SD & $7.17 \pm 6.10$ & $3.77 \pm 2.12^{b}$ & $32 \pm 5$ \\
\hline
\end{tabular}

Notes: ${ }^{\text {aPatients receiving Restasis }}{ }^{\circledR}$ (cyclosporine A) for $>6$ months. ${ }^{\mathrm{b}} \mathrm{P}=0.0212$ versus baseline, paired $t$-test.

Abbreviations: HLA-DR, human leukocyte antigen D-related; NA, not available; OD, oculus dexter; OS, oculus sinister; PEG, polyethylene glycol; PG, propylene glycol.

Use of some of these drugs has improved signs and symptoms of DED, but some have adverse effects, including pain, discomfort, and secondary ocular changes such as cataracts and glaucoma, all of which may limit their long-term use. ${ }^{28}$

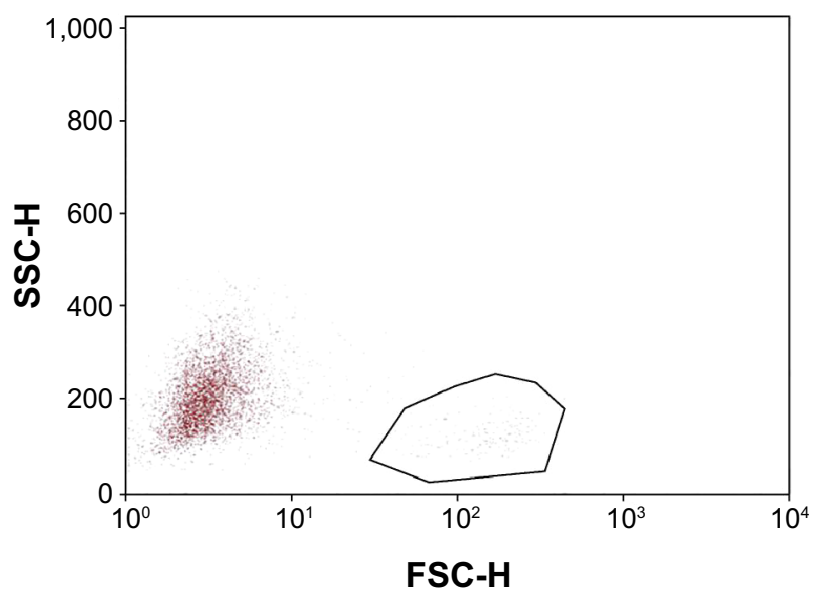

Figure 2 Representative dot plot of impression cytology samples collected before study treatment.

Notes: Samples were analyzed after cellular debris, aggregates, and naked antibodies were excluded. Cell size (forward scatter pulse height [FSC-H]) versus cellular granularity (side scatter pulse height [SSC-H]) of samples collected before the 30 day treatment period is plotted. The outlined area demonstrates the subpopulation of cells highly expressing human leukocyte antigen D-related antigen. In each sample, I,000-10,000 cells were analyzed. Samples containing less than I,000 cells were discarded. A masked observer examined all specimens.

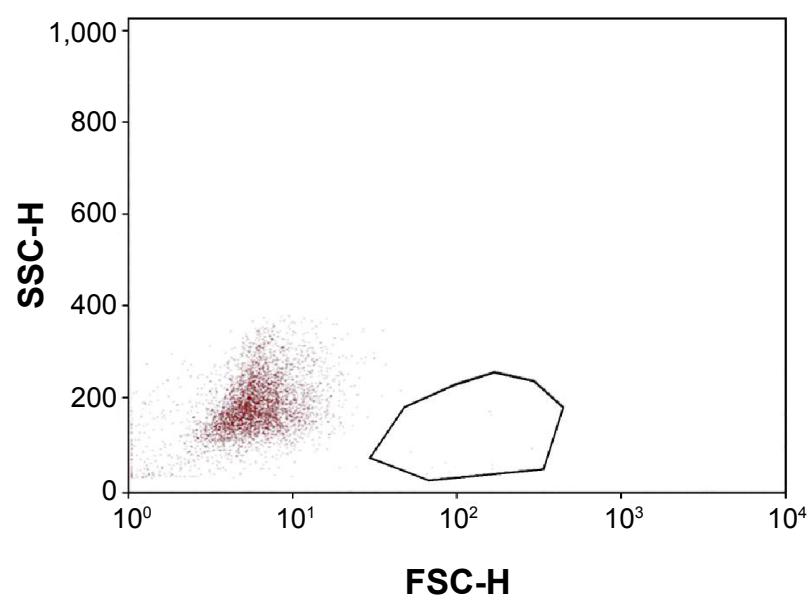

Figure 3 Representative dot plot of impression cytology samples collected after study treatment.

Notes: Samples were analyzed after cellular debris, aggregates, and naked antibodies were excluded. Cell size (forward scatter pulse height [FSC-H]) versus cellular granularity (side scatter pulse height $[\mathrm{SSC}-\mathrm{H}]$ ) of patient samples collected after 30 days of treatment is plotted. The outlined area demonstrates the subpopulation of cells highly expressing human leukocyte antigen D-related antigen. In each sample, I,000-10,000 cells were analyzed. Samples containing less than 1,000 cells were discarded. A masked observer examined all specimens.

With the use of the biomarker HLA-DR, therapeutic approaches to modulate inflammation associated with dry eye can indirectly be monitored. Sánchez et al performed a prospective, randomized study to assess the effect of an artificial tear eyedrop containing HP-Guar in DED after cataract surgery. ${ }^{19}$ This study randomized patients who had undergone cataract surgery into those receiving the usual postoperative eyedrops (antibiotic + steroid) and those receiving the same drops plus the artificial tear with HP-Guar. The following clinical assessments were evaluated: OSDI, surface staining, tear break-up time, and Schirmer scores, as well as laboratory analysis by flow cytometry to evaluate inflammatory markers and compared data from postoperative day 1 and postoperative day 30 . This study found significant differences in TFBUT, OSDI scores, and inflammatory markers HLA-DR and CD11b. Major histocompatibility complex HLA-DR is particularly relevant because it is normally expressed only by antigen-presenting cells but is expressed by ocular surface epithelial cells during immune-driven inflammation. In DED, immune-driven inflammation occurs when ocular surface epithelial cells express markers such as HLA-DR, which elicit further T-cell response, creating a cycle of inflammation and surface damage. Because of this, HLA-DR can be used to monitor inflammatory activity on the ocular surface and possibly the effectiveness of dry eye treatment.

Although studies have shown some pharmaceuticals increased tear production and decreased inflammation, patients with severe dry eyes find incomplete relief of 
symptoms and usually still need a supplemental use of an artificial tear product. ${ }^{29}$ The use of tear substitutes as part of the management of DED is a form of symptomatic approach to the treatment. Tear substitutes or artificial tears sometimes contain additional agents that increase their viscosity in order to improve patients' comfort and return the ocular surface and tear film to the normal homeostatic state. HP-Guar is used as a gelling or filming agent added to PEG/PG and has been suggested to preferentially bind to more desiccated or damaged areas of the surface of epithelial cells providing protection. ${ }^{30-32}$ When HP-Guar ( $\mathrm{pH}$ of 7.0) comes into contact with tears $(\mathrm{pH} 7.5-7.8)$, it forms a matrix with borate ions, and thus, the contact time with the PEG/PG lubricant is prolonged, which leads to a stabilization of the tear film. ${ }^{32}$ In rabbits, an exposure as short as 1 week could lead to an increase in precorneal mucous layer thickness. ${ }^{18}$ A study done by Sall et al showed that cyclosporine A (Restasis) combined with an artificial tear worked well in improving signs and symptoms of DED; however, not all artificial tears worked the same. ${ }^{33}$ They found that Restasis combined with a lubricant Systane ${ }^{\circledR}$ (Alcon) that contained PEG/PG and the gelling agent HP-Guar worked better than when combined with a preservative-free carboxymethylcellulose agent Refresh plus ${ }^{\circledR}$ (Allergan). Interestingly, Restasis combined with Systane compared to Systane alone did not show any statistically significant difference in the improvement of signs and symptoms. ${ }^{33}$

According to the DEWS report on the management and therapy of dry eye in 2007, although ocular lubricants have been shown to provide some protection of the ocular surface, and some improvement in patient symptoms and objective findings, they have not been demonstrated in controlled clinical trials to be sufficient to resolve ocular surface disorder and inflammation seen in most dry eye sufferers. ${ }^{34}$ Springs searched for peer-reviewed articles related to DED and PEG/ PG + HP-Guar and concluded that the drops would reduce corneal and conjunctival staining in patients diagnosed with this condition, improve tear film stability, have a low coefficient of friction in an in vitro model, and improve maintenance of best-corrected visual acuity over time. ${ }^{32}$ However, there was no mention of this preparation being effective in modulating inflammation associated with DED.

In our study, it was shown that following 30 continuous days of use with the PEG/PG + HP-Guar, there were statistically significant improvements in measurements of general ocular health. As part of the methodology, we also analyzed the effect of treatment on the ocular surface using impression cytology and used flow cytometry to monitor inflammatory markers associated with DED. We found that the use of PEG/PG + HP-Guar in our study protocol not only led to reductions of the total DEWS score but also improvement in OSDI score, corneal staining, and TFBUT. More than half of the patients showed decreased expression of HLA-DR after treatment with PEG/PG + HP-Guar. Not all patients showed the same magnitude of decrease in HLA-DR and some patients showed an increase in HLA-DR despite improvement in clinical signs and symptoms of DED. This may be due to individual variability in response to the treatment, or possibly, some may require more time to achieve a decrease in surface inflammation. Our masked analysis demonstrated that overall, the average percentage of HLA-DR-positive cells was significantly decreased on the ocular surface after 30 days of use of PEG/PG + HP-Guar $(P=0.02)$.

To our knowledge, this is the first reported evidence that an over-the-counter lubricant eyedrop solution can help decrease the immune response of the ocular surface in patients primarily diagnosed with DED, as evidenced by a reduction in a biomarker of ocular inflammation, percent positive HLA-DR cells. Similar results have been published by Sánchez et al, who demonstrated that the use of PEG/ PG + HP-Guar after phacoemulsification decreased dry eye parameters and inflammatory markers including HLA-DR; hence, the use of these drops might not only be an option for primary dry eye patients but also for dry eye or ocular surface diseases associated with ocular procedures. ${ }^{19}$ This demonstrates that impression cytology and flow cytometry may be considered a minimally invasive and objective means to monitor the disease process as well as treatment effects in DED. These results underscore the need for further, largescale studies concerning the use of artificial tears not only as a symptomatic treatment but also objectively as a therapy reducing parameters of disease activity.

This study had some limitations. The small sample size did not allow for multivariate testing for correlation of HLA-DR expression with other inflammatory markers. Further, the study was open-label with only one arm and no control group. The absence of a control group did not allow a comparison between treatment with the study formulation and no treatment, which somewhat limits the strength of conclusions that can be drawn regarding the effects of the study formulation. Also, two of the patients analyzed had been using an anti-inflammatory agent, cyclosporine A (Restasis) at the time of enrollment and there was no washout period. Because of the anti-inflammatory nature of Restasis, posttreatment decrease in inflammatory activity cannot be solely attributed to the test drug. Despite these limitations, our 
results demonstrated that HLA-DR expression significantly decreased from baseline, and many signs and symptoms of DED were significantly improved in patients with DED who received treatment with PEG/PG + HP-Guar, suggesting that patients may benefit from use of this artificial tear formulation.

\section{Conclusion}

We have shown that PEG/PG + HP-Guar is effective in improving signs and symptoms of ocular surface disease associated with dry eye. Interestingly, it was also shown that the inflammatory aspect of the disorder may be further modulated with the use of this lubricant eyedrop solution. This demonstrates that symptomatic treatment of DED that attempts to bring back the ocular surface to a homeostatic state can produce a corresponding decrease in the immune response of the eye to surface damage. Furthermore, because inflammation plays a pivotal role in DED, impression cytology and flow cytometry may be valuable tools in its diagnosis and treatment and provide an objective minimally invasive biomarker and metric to classify severity of ocular surface disease and to monitor response to treatment. This study may serve as a preliminary investigation of the role of artificial tears in regulating inflammatory response in DED. Future studies will include larger patient populations, longer follow-up durations, and comparison of PEG/PG + HP-Guar with other eyedrop formulations for dry eye. Additionally, characterization of the role of inflammatory markers in DED may contribute to improvements in diagnostic modalities and treatment monitoring of this disease.

\section{Acknowledgments}

This study was sponsored by Alcon Laboratories, Inc., and was funded, in part, by the Martin and Toni Sosnoff Fund.

\section{Disclosure}

The authors report no conflicts of interest in this work. The authors alone are responsible for the content and writing of the paper.

\section{References}

1. The definition and classification of dry eye disease: report of the definition and classification subcommittee of the International Dry Eye WorkShop (2007). Ocul Surf. 2007;5(2):75-92.

2. The epidemiology of dry eye disease: report of the epidemiology subcommittee of the International Dry Eye WorkShop (2007). Ocul Surf. 2007; 5(2):93-107.

3. Barabino S, Montaldo E, Solignani F, Valente C, Mingari MC, Rolando M. Immune response in the conjunctival epithelium of patients with dry eye. Exp Eye Res. 2010;91(4):524-529.
4. Massingale ML, Li X, Vallabhajosyula M, Chen D, Wei Y, Asbell PA. Analysis of inflammatory cytokines in the tears of dry eye patients. Cornea. 2009;28(9):1023-1027.

5. Rolando M, Barabino S, Mingari C, Moretti S, Giuffrida S, Calabria G. Distribution of conjunctival HLA-DR expression and the pathogenesis of damage in early dry eyes. Cornea. 2005;24(8):951-954.

6. Tsubota K, Fujihara T, Saito K, Takeuchi T. Conjunctival epithelium expression of HLA-DR in dry eye patients. Ophthalmologica. 1999;213(1): $16-19$.

7. Versura P, Profazio V, Schiavi C, Campos EC. Hyperosmolar stress upregulates HLA-DR expression in human conjunctival epithelium in dry eye patients and in vitro models. Invest Ophthalmol Vis Sci. 2011; 52(8):5488-5496.

8. Baudouin C, Brignole F, Becquet F, Pisella PJ, Goguel A. Flow cytometry in impression cytology specimens. A new method for evaluation of conjunctival inflammation. Invest Ophthalmol Vis Sci. 1997;38(7): $1458-1464$.

9. Baudouin C, Brignole F, Pisella PJ, De Jean MS, Goguel A. Flow cytometric analysis of the inflammatory marker HLA DR in dry eye syndrome: results from 12 months of randomized treatment with topical cyclosporin A. Adv Exp Med Biol. 2002;506(pt B):761-769.

10. Brignole F, De Saint-Jean M, Goldschild M, Becquet F, Goguel A, Baudouin C. Expression of Fas-Fas ligand antigens and apoptotic marker APO2.7 by the human conjunctival epithelium. Positive correlation with class II HLA DR expression in inflammatory ocular surface disorders. Exp Eye Res. 1998;67(6):687-697.

11. Brignole F, Pisella PJ, Goldschild M, De Saint Jean M, Goguel A, Baudouin C. Flow cytometric analysis of inflammatory markers in conjunctival epithelial cells of patients with dry eyes. Invest Ophthalmol Vis Sci. 2000;41(6):1356-1363.

12. Lopin E, Deveney T, Asbell PA. Impression cytology: recent advances and applications in dry eye disease. Ocul Surf. 2009;7(2):93-110.

13. Mrugacz M,Zak J, Bakunowicz-Lazarczyk A, Wysocka J, Minarowska A. Flow cytometric analysis of HLA-DR antigen in conjunctival epithelial cells of patients with cystic fibrosis. Eye (Lond). 2007;21(8): 1062-1066.

14. Pisella PJ, Brignole F, Debbasch C, et al. Flow cytometric analysis of conjunctival epithelium in ocular rosacea and keratoconjunctivitis sicca. Ophthalmology. 2000;107(10):1841-1849.

15. Research in dry eye: report of the research subcommittee of the International Dry Eye WorkShop (2007). Ocul Surf. 2007;5(2):179-193.

16. Liang H, Brignole-Baudouin F, Pauly A, Riancho L, Baudouin C. Polyquad-preserved travoprost/timolol, benzalkonium chloride (BAK)-preserved travoprost/timolol, and latanoprost/timolol in fixed combinations: a rabbit ocular surface study. Adv Ther. 2011;28(4): 311-325.

17. Lopez Bernal D, Ubels JL. Quantitative evaluation of the corneal epithelial barrier: effect of artificial tears and preservatives. Curr Eye Res. 1991; 10(7):645-656.

18. Moon SW, Hwang JH, Chung SH, Nam KH. The impact of artificial tears containing hydroxypropyl guar on mucous layer. Cornea. 2010; 29(12):1430-1435.

19. Sánchez MA, Arriola-Villalobos P, Torralbo-Jiménez P, et al. The effect of preservative-free HP-Guar on dry eye after phacoemulsification: a flow cytometric study. Eye (Lond). 2010;24(8):1331-1337.

20. Lavappa KS, Macy ML, Shannon JE. Examination of ATCC stocks for HeLa marker chromosomes in human cell lines. Nature. 1976;259(5540): 211-213.

21. Lavappa KS. Survey of ATCC stocks of human cell lines for HeLa contamination. In Vitro. 1978;14(5):469-475.

22. De Saint Jean M, Baudouin C, Di Nolfo M, et al. Comparison of morphological and functional characteristics of primary-cultured human conjunctival epithelium and of Wong-Kilbourne derivative of Chang conjunctival cell line. Exp Eye Res. 2004;78(2):257-274.

23. Jester JV, Ho-Chang J. Modulation of cultured corneal keratocyte phenotype by growth factors/cytokines control in vitro contractility and extracellular matrix contraction. Exp Eye Res. 2003;77(5):581-592. 
24. Methodologies to diagnose and monitor dry eye disease: report of the diagnostic methodology subcommittee of the International Dry Eye WorkShop (2007). Ocul Surf. 2007;5(2):108-152.

25. Bron AJ, Evans VE, Smith JA. Grading of corneal and conjunctival staining in the context of other dry eye tests. Cornea. 2003;22(7):640-650.

26. Lemp MA. Report of the National Eye Institute/Industry workshop on clinical trials in dry eyes. CLAO J. 1995;21(4):221-232.

27. Stevenson D, Tauber J, Reis BL. Efficacy and safety of cyclosporin A ophthalmic emulsion in the treatment of moderate-to-severe dry eye disease: a dose-ranging, randomized trial. The Cyclosporin A Phase 2 Study Group. Ophthalmology. 2000;107(5):967-974.

28. Asbell PA, Spiegel S. Ophthalmologist perceptions regarding treatment of moderate to severe dry eye: results of a physician survey. Trans Am Ophthalmol Soc. 2009;107:205-210.

29. Perry HD, Solomon R, Donnenfeld ED, et al. Evaluation of topical cyclosporine for the treatment of dry eye disease. Arch Ophthalmol. 2008;126(8):1046-1050.
30. Cervan-Lopez I, Saenz-Frances-San-Baldomero F, Benitez-DelCastillo JM, Garcia-Sanchez J. [Reduction of corneal permeability in patients treated with HP-guar: a fluorophotometric study]. Arch Soc Esp Oftalmol. 2006;81(6):327-332.

31. Labbé A, Pauly A, Liang H, et al. Comparison of toxicological profiles of benzalkonium chloride and polyquaternium-1: an experimental study. J Ocul Pharmacol Ther. 2006;22(4):267-278.

32. Springs CL. Novel hydroxypropyl-guar gellable lubricant eye drops for treatment of dry eye. Adv Ther. 2010;27(10):681-690.

33. Sall KN, Cohen SM, Christensen MT, Stein JM. An evaluation of the efficacy of a cyclosporine-based dry eye therapy when used with marketed artificial tears as supportive therapy in dry eye. Eye Contact Lens. 2006;32(1):21-26.

34. Management and therapy of dry eye disease: report of the management and therapy subcommittee of the International Dry Eye WorkShop (2007). Ocul Surf. 2007;5(2):163-178.
Clinical Ophthalmology

\section{Publish your work in this journal}

Clinical Ophthalmology is an international, peer-reviewed journa covering all subspecialties within ophthalmology. Key topics include: Optometry; Visual science; Pharmacology and drug therapy in eye diseases; Basic Sciences; Primary and Secondary eye care; Patient Safety and Quality of Care Improvements. This journal is indexed on

Submit your manuscript here: http://www.dovepress.com/clinical-ophthalmology-journal

\section{Dovepress}

PubMed Central and CAS, and is the official journal of The Society of Clinical Ophthalmology (SCO). The manuscript management system is completely online and includes a very quick and fair peer-review system, which is all easy to use. Visit http://www.dovepress.com/ testimonials.php to read real quotes from published authors. 